\title{
Does a text messaging intervention improve knowledge, attitudes and practice regarding iodine deficiency and iodized salt consumption?
}

\author{
Ladan Mehran ${ }^{1}$, Pantea Nazeri ${ }^{2}$, Hossein Delshad' ${ }^{1}$ Parvin Mirmiran², \\ Yadollah Mehrabi ${ }^{3}$ and Fereidoun Azizi ${ }^{1, *}$ \\ ${ }^{1}$ Endocrine Research Center, Research Institute for Endocrine Sciences, Shahid Beheshti University of Medical \\ Sciences, PO Box 19395-4763, Tehran, Islamic Republic of Iran: ${ }^{2}$ Obesity Research Center, Research Institute \\ for Endocrine Sciences, Shahid Beheshti University of Medical Sciences, Tehran, Islamic Republic of Iran: \\ ${ }^{3}$ Department of Epidemiology, School of Public Health, Shahid Beheshti University of Medical Sciences, Tehran, \\ Islamic Republic of Iran
}

Submitted 18 August 2011: Final revision received 4 January 2012: Accepted 3 February 2012: First published online 2 July 2012

\begin{abstract}
Objective: To determine the effectiveness of implementation of a prevention programme via mobile phone text messaging in enhancing knowledge, attitudes and practice concerning iodine deficiency and iodized salt consumption.

Design: In a randomized controlled trial, participants were subjected to a brief tele-educational support regarding iodine deficiency and the importance of iodized salt consumption. The intervention group received daily text messages via mobile phone for 6 weeks. Knowledge, attitude and practice scores, urinary iodine concentration and salt iodine content were assessed at baseline and 8 weeks after the intervention.

Setting: Participants were recruited from health-care centres in Tehran, the capital city of Iran.

Subjects: For the present study 205 females aged $\geq 18$ years were randomly assigned to the intervention ( $n$ 95) and control ( $n$ 110) groups.

Results: A significant difference was found in median knowledge scores between the intervention and control groups at follow-up $(P=0 \cdot 004)$. There was also a significant difference in median attitude scores between the intervention and control groups $(P=0 \cdot 02)$. The intervention group did not differ significantly in median practice score, urinary iodine concentration and salt iodine content from the control group.

Conclusions: Text messaging interventions are effective in improving individuals' knowledge and attitudes regarding preventive health-care topics.
\end{abstract}

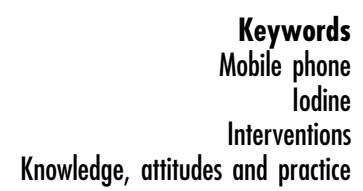

Iodine deficiency, due to its effects on the developing brain, especially at critical stages during pregnancy and early childhood, imposes a high financial and social burden on the governments of countries affected by iodine-deficiency disorders ${ }^{(1,2)}$. Over the last two decades, many countries have implemented salt iodization programmes as the best preventive strategy and achieved great progress in controlling iodine deficiency ${ }^{(3)}$. Monitoring the sustainability of the programme is of utmost importance $^{(4,5)}$.

Although Iran was declared to be free of iodinedeficiency disorders in the year $2000^{(6)}$, results of the last national survey in 2007 and another study by Nazeri et al. in Tehran were indicative of decreases in median urinary iodine concentrations and salt iodine levels in comparison to previous reports ${ }^{(7)}$. The public health education campaign regarding iodized salt consumption was conducted through the earlier years of implementation of the universal salt iodization programme from 1989 to 1994; after that there has been little or no public knowledge dissemination regarding iodized salt consumption via various mass media, measures which are essential to raise levels of public awareness and need to be prioritized in health policies.

Recent studies have focused on the effectiveness of health behaviour modification programmes via telecommunication $^{(8-10)}$ and the Internet ${ }^{(11)}$. Nowadays mobile phones and text messaging have been integrated into all aspects of communities in both developed and developing countries. Short message services (SMS) allow for instant delivery of messages whenever or wherever the person is located; it is also an inexpensive means of 
intervention for health programmes. The usage of mobile phones and SMS has spread through all age groups with different cultural and socio-economic backgrounds; contrary to commonly held belief, the use of mobile phones is more prevalent in younger adults with lower socio-economic, educational and poorer health status ${ }^{(12)}$. In Iran mobile phone usage is extremely common and occurs in all classes of society; in particular housewives use mobile phones. Iran, with a population of 75 million, has about 68 million mobile phone subscribers and mobile phone networks offer coverage to all cities and to approximately $93 \%$ of the population. Iranians send about 80 million SMS per day ${ }^{(13)}$.

These new technologies have great potential for promoting public health by delivering interventions and interactive communication and assisting people in disease self-management and prevention ${ }^{(14-16)}$. SMS interventions have found to be effective in improving patients' attendance in clinics and primary care ${ }^{(17)}$, the coordination of medical staff ${ }^{(18)}$ and increasing adherence to medical therapy ${ }^{(19,20)}$. Most studies on informational interventions delivered by mobile phones via SMS or voice features have achieved positive results in preventive health behavioural change concerning physical activity ${ }^{(21)}$, weight loss control $^{(22)}$ and smoking cessation ${ }^{(23,24)}$, and in clinical care, especially for self-management of chronic diseases such as diabetes $^{(25,26)}$, asthma ${ }^{(27)}$ and $\mathrm{CHD}^{(28)}$.

It is an ongoing challenge to find more effective and convenient approaches for providing care and preventive management support that lead to behavioural change and better health outcomes. To our knowledge no randomized trial has evaluated the effect of mobile texting in promoting health behaviour regarding the prevention of iodine deficiency. The present study was designed to evaluate the role of an intervention implemented using mobile phone text messaging in enhancing the knowledge, attitudes and practice of individuals concerning the importance of iodized salt consumption.

\section{Experimental methods}

\section{Participants and study design}

In the present randomized, controlled trial, 205 females were recruited from health-care centres in Tehran, the capital city of Iran. Inclusion criteria were age over 18 years, housewives responsible for cooking, having a mobile phone with text message feature and the ability and willingness to read and retrieve text messages; the exclusion criteria were pregnancy and lactation. All participants received brief information regarding the importance of iodized salt consumption via fixed-line telephone at baseline. Participants were then randomly assigned into the intervention ( $n$ 95) or control ( $n$ 110) group. Personal information, including personal history of thyroid diseases and hypertension, consumption of medications and iodine-containing supplements, were obtained and documented using an interviewer-administered questionnaire.

Participants in the intervention group received daily text messages via their mobile phone for 6 weeks. The text messages consisted of information regarding the definition and consequences of iodine deficiency in individuals and the importance of consumption and proper storage of iodized salt as the best preventive approach. Follow-up evaluation was conducted 2 weeks after the end of the intervention. At baseline and follow-up, salt and urine samples were obtained from each of the participants in the control and intervention groups and questionnaires on knowledge, attitudes and practice regarding iodine deficiency and iodized salt were also completed. Salt samples (2 tablespoons) were collected from the participants' households, kept in lightproof and closed plastic cans and labelled with the code of participants in each group. Urine samples were collected and transferred in screw-topped labelled plastic vials to the iodine laboratory of the Endocrine Research Center at Shahid Beheshti University of Medical Sciences, the Reference Laboratory for the Eastern Mediterranean Region. The aliquots were kept frozen until iodine measurement.

Scores for knowledge, attitudes and practice were assessed by questionnaires and face-to-face interviews. Knowledge questions included yes/no/don't know and true/false/don't know questions (ten questions, each scored as yes/true $=3$ points, don't know $=2$ points and no/false $=1$ point). Attitudes (eight questions) were assessed based on Likert criteria from 1 point as the weakest to 5 points as a desirable score. Questions related to practices included iodized salt consumption, conditions of iodized salt storage (e.g. exposure to light, humidity and uncovered containers) and time of salt addition during the cooking process (six questions, each scoring 1 point).

For face validity, ten women completed a questionnaire which assessed their awareness levels on the importance of iodine consumption in their daily lives. For content validity, an expert panel of ten specialists in health education, endocrinology, nutrition and sociology examined the initial questionnaire. The panel was asked to comment on individual items in relation to the accuracy and style; items were modified slightly based on their expert reviews. In addition, Cronbach's $\alpha$ was determined for internal consistency of the questionnaire (Cronbach's $\alpha=0 \cdot 83$ ). Informed consent was obtained from the participants of each group.

\section{Laboratory measurements}

Iodine content of salt samples was determined using the iodometric titration method with a sensitivity of $1 \mathrm{ppm}$ and $\mathrm{CV}$ of $1 \%$. The values obtained are reported in ppm. Iodine concentrations in urine samples were analysed using the Sandell-Kolthoff (acid digestion) reaction and 
results are expressed as $\mu \mathrm{g}$ iodine/1 urine. The intra- and inter-assay CV were $9 \cdot 6 \%$ and $10 \cdot 4 \%$, respectively, and the sensitivity was $2 \mu \mathrm{g} / \mathrm{l}$.

\section{Statistical analyses}

Statistical analyses were done using the SPSS for Windows statistical software package version 15.0 (2006; SPSS Inc., Chicago, IL, USA), $P<0.05$ being considered significant. Qualitative variables in baseline characteristics are presented as frequency distribution (\%), while mean and standard deviation are used for age as a continuous numerical variable. Normality of the variables was assessed by the Kolmogorov-Smirnov test. Depending on normality of variables, categorical variables were compared by Mann-Whitney or $\chi^{2}$ tests. The Wilcoxon test was used to compare baseline and follow-up variables within each group, whereas the changes in each variable between the two groups at follow-up were compared using the Mann-Whitney test. Spearman correlation was applied to show the correlation between education levels and any of the outcome variables at baseline.

\section{Results}

The basic characteristics of intervention and control groups are given in Table 1 . There was no difference in

Table 1 Baseline characteristics of participants according to control and intervention status, females aged $\geq 18$ years, Tehran, Iran

\begin{tabular}{lcrc}
\hline & $\begin{array}{c}\text { Intervention } \\
\text { group } \\
(n \text { 95) }\end{array}$ & $\begin{array}{c}\text { Control } \\
\text { group } \\
(n \text { 110) }\end{array}$ & $P$ value \\
Characteristic & & & 0.730 \\
Education (\%) & 89.5 & 90.9 & \\
$\quad$ DDiploma & 10.5 & 9.1 & \\
>Diploma & 5.3 & 3.6 & 0.571 \\
Goitre (\%) & 7.4 & 5.5 & 0.575 \\
Hypothyroidism (\%) & 0.0 & 0.9 & 0.352 \\
Hyperthyroidism (\%) & 9.5 & 14.5 & 0.268 \\
Hypertension (\%) & 6.3 & 4.5 & 0.575 \\
Use of iodine-containing & & & \\
$\quad$ supplement (\%) & & & \\
\hline
\end{tabular}

mean age between the intervention and control groups (44.5 (SD 9.9) years $v$. 44.4 (SD 10.9) years; $P=0.922$ ). Educational status in both the intervention and control groups did not differ significantly. No significant difference was found in the history of any thyroid disorders, hypertension and the use of iodine-containing supplements, factors which may interfere with participants' iodine status (Table 1$)$. In addition, knowledge $(P=0 \cdot 826)$, attitude $(P=0 \cdot 933)$ and practice scores $(P=0 \cdot 072)$, urinary iodine concentrations $(P=0 \cdot 959)$ and salt iodine contents $(P=0.793)$ not differ significantly between the two groups at baseline.

The baseline and follow-up values for of salt iodine content, urinary iodine concentration and knowledge, attitude and practice scores in the intervention and control groups are shown in Table 2 . At follow-up the intervention group had significant increases in all variables; $25 \cdot 3 \%$ in median urinary iodine concentration (from 79 to $99 \mu \mathrm{g} / \mathrm{l}$; $P=0.001$ ), $49 \cdot 5 \%$ in salt iodine content (from $21 \cdot 2$ to $31.7 \mathrm{ppm} ; P<0.001$ ), $30.0 \%$ in knowledge score (from 20 to 26 points out of a total of 30 points; $P<0 \cdot 001$ ), $5 \cdot 8 \%$ in attitude score (from 34 to 36 points out of a total of 40 points; $P<0.001$ ) and $20.0 \%$ in practice score (from 5 to 6 points out of a total of 6 points; $P<0 \cdot 001$ ).

In the control group, there was $12 \cdot 5 \%$ improvement in median urinary iodine concentration (from 77.5 to $87 \cdot 2 \mu \mathrm{g} / \mathrm{l}), 57 \cdot 7 \%$ improvement in salt iodine content (from $20 \cdot 1$ to $31 \cdot 7 \mathrm{ppm}$ ), $15 \cdot 0 \%$ improvement in knowledge (from 20 to 23 points out of a total of 30 points) and $25.0 \%$ improvement in practice scores (from 4 to 5 points out of a total of 6 points). The differences between baseline and follow-up in the control group were significant only for salt iodine content $(P<0 \cdot 001)$, knowledge $(P=0.035)$ and practice scores $(P<0 \cdot 001)$. There was no change in the attitude score $(P=0.705)$ and median urinary iodine concentration $(P=0 \cdot 137)$ in the control group before and after the intervention.

A significant difference was found in knowledge scores between the intervention (median $=26$; interquartile range $(\mathrm{IQR}) 21,28)$ and control $($ median $=23$; IQR 16, 27)

Table 2 Salt iodine content, urinary iodine concentration and knowledge, attitude and practice scores in the control and intervention groups at baseline and follow-up

\begin{tabular}{|c|c|c|c|c|c|c|c|c|c|}
\hline & \multicolumn{4}{|c|}{ Intervention group } & \multicolumn{4}{|c|}{ Control group } & \multirow[b]{3}{*}{$\begin{array}{c}P \text { for } \\
\text { SMS effect* }\end{array}$} \\
\hline & \multicolumn{2}{|c|}{ Baseline } & \multicolumn{2}{|c|}{ Follow-up } & \multicolumn{2}{|c|}{ Baseline } & \multicolumn{2}{|c|}{ Follow-up } & \\
\hline & Median & IQR & Median & IQR & Median & IQR & Median & IQR & \\
\hline Salt iodine content (ppm) & $21 \cdot 2$ & $3 \cdot 2,31 \cdot 7$ & $31 \cdot 7 t$ & $21 \cdot 2,38 \cdot 1$ & $20 \cdot 1$ & $2 \cdot 1,31 \cdot 7$ & $31 \cdot 7 t$ & $20 \cdot 1,38 \cdot 1$ & 0.576 \\
\hline Urinary iodine concentration $(\mu \mathrm{g} / \mathrm{l})$ & $79 \cdot 0$ & $46 \cdot 0,127 \cdot 1$ & $99 \cdot 0+$ & $54 \cdot 0,215 \cdot 0$ & $77 \cdot 5$ & $36 \cdot 5,132 \cdot 1$ & $87 \cdot 2$ & $51 \cdot 7,152 \cdot 0$ & $0 \cdot 144$ \\
\hline Knowledge score & 20 & 16,23 & $26+\neq$ & 21,28 & 20 & 15,24 & $23+$ & 16,27 & 0.004 \\
\hline Attitude score & 34 & 32,36 & $36 t, \ddagger$ & 33,39 & 34 & 32,37 & 34 & 31,37 & 0.027 \\
\hline Practice score & 5 & 4,5 & $6+$ & 5,6 & 4 & 4,5 & $5+$ & 4,6 & 0.395 \\
\hline
\end{tabular}

IQR, interquartile range; SMS, short message services.

${ }^{*} P$ for the difference in variables between the intervention and control groups.

tMedian value was significantly different from that at baseline $(P<0.05)$.

¥Median value was significantly different from that of the control group $(P<0.05)$. 
groups at follow-up $(P=0 \cdot 004)$. There was also significant difference in attitude scores between the intervention $($ median $=36 ; \mathrm{IQR} 33,39)$ and control (median $=34$; IQR $31,37)$ groups $(P=0 \cdot 027)$. Compared with the baseline values, at follow-up, text messaging was associated with a twofold higher increase in median urinary iodine concentration in the intervention group $(20.0 \mu \mathrm{g} / 1$ increase, from 79 to $99 \mu \mathrm{g} / \mathrm{l})$ compared with the controls $(9 \cdot 7 \mu \mathrm{g} / \mathrm{l}$ increase, from 77.5 to $87 \cdot 2 \mu \mathrm{g} / \mathrm{l}$ ); however, the difference was not statistically significant $(P=0 \cdot 144)$.

\section{Discussion}

The present trial evaluated for the first time the effect of a text messaging educational programme in enhancing iodine deficiency-related knowledge, attitude, practice and outcome measures including salt iodine content and urinary iodine concentration. We found that the text messaging intervention had a significant positive impact, especially on knowledge and attitudes; however, no significant positive changes were observed in behaviour as reflected by practice score, salt iodine content and urinary iodine concentrations.

At follow-up, significant positive trends were demonstrated in knowledge, attitude and practice scores and related outcome measures (urinary and salt iodine levels) within the intervention group; however, the intervention group did not differ significantly from controls in behaviour as reflected by practice scores and outcome measures (salt and urinary iodine levels), indicating that the isolated impact of text messaging on practice was not significant. This was due to significant increases in practice score and salt iodine content and the non-significant, but remarkable, improvement found in median urinary iodine concentration in the control group at follow-up, which may have been due to the baseline telecommunication intervention (via landline telephone) or the possibility of other educational modalities; however, we did not specifically track any possible confounders. In addition some news regarding the production and distribution of non-iodized or less adequately iodized salt before the trial might have prompted salt factories to produce more standardized iodized salt and improvement of salt iodine content in the market during followup period could act as a confounder ${ }^{(7)}$.

Health-care education via mobile phone offers a great opportunity for designing and developing healthcare interventions in order to help individuals in disease prevention and self-management; this educational modality, which may improve health outcomes, is relatively inexpensive, flexible, individually tailored and easily deliverable across extensive geographical regions, reaching a wide population, especially those with lower socio-economic status $^{(12)}$. A few systematic reviews have reported positive behavioural trends through SMS-based interventions ${ }^{(14-16,29)}$; however, the evidence documented is inconclusive due to methodological limitations and variations in study designs, settings, populations, length of intervention, SMS delivery intensity, sample size and outcome measures. Text messaging demonstrated only early efficacy in most studies and its long-term efficacy remains to be determined. In addition, it is difficult to determine the relative impact of text messaging strategies in some studies as SMS is used as an adjunct to other delivery means of intervention, e.g. Internet or face-to-face communication, voice calls, etc., rather than a comprehensive strategy $^{(14,15)}$. Two systematic reviews on randomized controlled trials provide positive results in applying text messaging alone as a primary medium for behavioural change in disease prevention and management, to isolate the effectiveness of text messaging ${ }^{(16,29)}$. Most studies have focused on clinical care interventions via SMS to increase disease self-management and adherence to a treatment programme in sick individuals with chronic diseases such as diabetes ${ }^{(15,25,26)}$, asthma ${ }^{(27)}, \mathrm{CHD}^{(28)}$ and immune deficiency ${ }^{(19)}$. A few studies have been conducted on preventive interventions to promote health behaviour in healthy individuals via SMS, including studies on smoking cessation ${ }^{(23,30)}$, physical activity ${ }^{(21,31)}$, weight control $^{(22,32,33)}$, sexual health ${ }^{(34)}$ and sunscreen use $^{(35)}$; most of these showed positive results regarding the mobile phone text messing intervention.

The main shortcomings of the present trial lie in the small sample size, low validity of the questionnaire especially on the practice scale, lack of generalizability due to recruiting only females, the possibility of other interfering confounders during the follow-up period and not evaluating the long-term efficacy of the intervention. The trial's strengths are its randomized controlled design, carefully designed content of text messaging, relying on both subjective and objective measures to assess intervention effects on targeted behaviour (a major bias in many similar studies), and the presenting of a novel issue of high concern and with international applicability for designing future strategies.

The present study revealed the efficacy of an intervention delivered by text messaging in improving knowledge and attitudes, but not behaviour, of the study population. There is still much to be learned about optimizing and integrating this new intervention method into clinical practice and health-care systems. Monitoring the maintenance of the behavioural effect after the intervention should be also considered in future research. In order to gain greater insight into the effectiveness of mobile phone-based interventions in improving health care, further trials are warranted in different geographical and cultural settings, on larger sample sizes and more representative populations, using more valid questionnaires on various types of health care, medical or nutritional topics, especially on preventive strategies like behavioural modifications regarding consumption of iodized salt. 
Iodine deficiency is the major cause of preventable mental retardation and salt iodization is the best strategy to combat it. Mobile phone text messaging is a convenient, low-cost and practical method of education which increases public knowledge regarding iodized salt consumption and has the added flexibility that it can be used in all locations, in particular in low-income countries with insufficient iodine nutrition.

\section{Acknowledgements}

The study was supported by financial grants from the Research Institute for Endocrine Sciences, Shahid Beheshti University of Medical Sciences, Tehran, Iran. None of the authors had any personal or financial conflicts of interest. L.M. contributed to the design and supervision of the study, data analysis and writing of the manuscript; P.N. contributed to the performance of the study, data analysis and writing of the manuscript; H.D. contributed to the design of the study and writing of the manuscript; P.M. contributed to the design of the study and writing of the manuscript; Y.M. contributed to the statistical analysis and writing of the manuscript; F.A. contributed to the design of the study and writing, reading and final approval of the manuscript. The authors sincerely acknowledge all of the field investigators for their assistance and Ms Niloufar Shiva for language editing of the manuscript.

\section{References}

1. Delange FM \& Dunn JT (2005) Iodine deficiency. In The Thyroid: A Fundamental and Clinical Text, pp. 264-288 [LE Braverman and RD Utiger, editors]. Philadelphia, PA: Lippincott Williams \& Wilkins.

2. Delange F (2001) Iodine deficiency as a cause of brain damage. Postgrad Med J 77, 217-220.

3. Hetzel BS (2002) Eliminating iodine deficiency disorders the role of the International Council in the global partnership. Bull World Health Organ 80, 410-413.

4. Zimmermann MB, Wegmuller R, Zeder C et al. (2004) Rapid relapse of thyroid dysfunction and goiter in school-age children after discontinuation of salt iodization. Am J Clin Nutr 79, 642-645.

5. UNICEF (2007) The State of The World's Children, 2008. Child Survival. New York: UNICEF.

6. Azizi F, Mehran L, Sheikholeslam R et al. (2008) Sustainability of a well-monitored salt iodization program in Iran: marked reduction in goiter prevalence and eventual normalization of urinary iodine concentrations without alteration in iodine content of salt. J Endocrinol Invest 31, 422-431.

7. Nazeri P, Mirmiran P, Mehrabi Y et al. (2010) Evaluation of iodine nutritional status in Tehran, Iran: iodine deficiency within iodine sufficiency. Thyroid 20, 1399-1406.

8. Chaudhry SI, Phillips CO, Stewart SS et al. (2007) Telemonitoring for patients with chronic heart failure: a systematic review. J Card Fail 13, 56-62.

9. Eakin EG, Lawler SP, Vandelanotte C et al. (2007) Telephone interventions for physical activity and dietary behavior change: a systematic review. Am J Prev Med 32, 419-434.
10. Pare G, Jaana M \& Sicotte C (2007) Systematic review of home telemonitoring for chronic diseases: the evidence base. J Am Med Inform Assoc 14, 269-277.

11. Webb TL, Joseph J, Yardley L et al. (2010) Using the internet to promote health behavior change: a systematic review and meta-analysis of the impact of theoretical basis, use of behavior change techniques, and mode of delivery on efficacy. J Med Internet Res 12, e4.

12. Koivusilta LK, Lintonen TP \& Rimpela AH (2007) Orientations in adolescent use of information and communication technology: a digital divide by sociodemographic background, educational career, and health. Scand J Public Health 35, 95-103.

13. National Portal for Electronic Services of Iran (2010) Iran has about 103 million mobile subscribers. http://en.iran. ir/en_news/news/774-iran-has-about-103-million-mobilesubscribers-.pdf (accessed December 2010).

14. Fjeldsoe BS, Marshall AL \& Miller YD (2009) Behavior change interventions delivered by mobile telephone shortmessage service. Am J Prev Med 36, 165-173.

15. Krishna S, Boren SA \& Balas EA (2009) Healthcare via cell phones: a systematic review. Telemed J E Health 15, 231-240.

16. Cole-Lewis H \& Kershaw T (2010) Text messaging as a tool for behavior change in disease prevention and management. Epidemiol Rev 32, 56-69.

17. Downer SR, Meara JG, Da Costa AC et al. (2006) SMS text messaging improves outpatient attendance. Aust Health Rev 30, 389-396.

18. Sherry E, Colloridi B \& Warnke PH (2002) Short message service (SMS): a useful communication tool for surgeons. ANZ J Surg 72, 369.

19. Kelly JD \& Giordano TP (2011) Mobile phone technologies improve adherence to antiretroviral treatment in a resource-limited setting: a randomized controlled trial of text message reminders. AIDS 25, 1137; reply 1138-1139.

20. Lester RT, Mills EJ, Kariri A et al. (2009) The HAART cell phone adherence trial (WelTel Kenya1): a randomized controlled trial protocol. Trials 10, 87.

21. Hurling R, Catt M, Boni MD et al. (2007) Using internet and mobile phone technology to deliver an automated physical activity program: randomized controlled trial. J Med Internet Res 9 , e7.

22. Joo NS \& Kim BT (2007) Mobile phone short message service messaging for behaviour modification in a communitybased weight control programme in Korea. J Telemed Telecare 13, 416-420.

23. Rodgers A, Corbett T, Bramley D et al. (2005) Do u smoke after txt? Results of a randomised trial of smoking cessation using mobile phone text messaging. Tob Control 14, 255-261.

24. Whittaker R, Borland R, Bullen C et al. (2009) Mobile phone-based interventions for smoking cessation. Cochrane Database Syst Rev issue 4, CD006611.

25. Franklin VL, Greene A, Waller A et al. (2008) Patients' engagement with 'Sweet Talk' - a text messaging support system for young people with diabetes. J Med Internet Res 10, e20.

26. Krishna S \& Boren SA (2008) Diabetes self-management care via cell phone: a systematic review. J Diabetes Sci Technol 2, 509-517.

27. Prabhakaran L, Chee WY, Chua KC et al. (2010) The use of text messaging to improve asthma control: a pilot study using the mobile phone short messaging service (SMS). J Telemed Telecare 16, 286-290.

28. Neubeck L, Redfern J, Fernandez R et al. (2009) Telehealth interventions for the secondary prevention of coronary heart disease: a systematic review. Eur J Cardiovasc Prev Rehabil 16, 281-289.

29. Wei J, Hollin I \& Kachnowski S (2011) A review of the use of mobile phone text messaging in clinical and healthy behaviour interventions. $J$ Telemed Telecare 17, 41-48. 
30. Obermayer JL, Riley WT, Asif O et al. (2004) College smoking-cessation using cell phone text messaging. J Am Coll Health 53, 71-78.

31. Newton KH, Wiltshire EJ \& Elley CR (2009) Pedometers and text messaging to increase physical activity: randomized controlled trial of adolescents with type 1 diabetes. Diabetes Care 32, 813-815.

32. Haapala I, Barengo NC, Biggs S et al. (2009) Weight loss by mobile phone: a 1-year effectiveness study. Public Health Nutr 12, 2382-2391.
33. Patrick K, Raab F, Adams MA et al. (2009) A text messagebased intervention for weight loss: randomized controlled trial. J Med Internet Res 11, e1.

34. Lim MS, Hocking JS, Hellard ME et al. (2008) SMS STI: a review of the uses of mobile phone text messaging in sexual health. Int J STD AIDS 19, 287-290.

35. Armstrong AW, Watson AJ, Makredes M et al. (2009) Text-message reminders to improve sunscreen use: a randomized, controlled trial using electronic monitoring. Arch Dermatol 145, 1230-1236. 